\title{
Electronic Circuit PHM with No Data
}

\author{
Varun Khemani, Dr. Michael. H. Azarian, Dr. Michael G. Pecht \\ Center for Advanced Life Cycle Engineering (CALCE) \\ University of Maryland \\ College Park, MD 20742 \\ vkheman@umd.edu
}

\begin{abstract}
Operational data from the target system is widely considered a pre-requisite for implementation of PHM, as it used as training data. Often this data is not available to PHM practitioners because health monitoring capabilities may not be installed in legacy systems unless there is a guarantee that a PHM framework will be viable/profitable. This research presents an approach in which PHM can be implemented without any operational data and is generic enough to be applied to any electronic circuit provided a simulation model of the system with acceptable fidelity can be developed. The research also introduces the Space-Filling Design, which can be used to generate the training data in a systematic, statistically valid framework, and is especially valuable for complex circuit with a large number of components. This design provides sufficient coverage of the parametric design space to be representative of the unavailable operational data, as well as incorporating the effects of parameter interaction on the simulated response of the system. Most PHM studies in literature ignore the effect of the degradation of interacting components. We show, how such an assumption can lead to incorrect fault diagnosis/RUL estimation and propose methods to screen for two-way and higher order interactions. Finally, we use various deep learning approaches in conjunction with physics-of-failure models for individual components to diagnose circuit faults and estimate circuit RUL. This simulation-based fusion prognostics approach is a holistic framework for all types of electronic circuits.
\end{abstract}

\section{PROBLEM STATEMENT}

Electronic circuits are a vital in the reliability and availability of industrial systems. Unexpected circuit failures in such systems can have severe implications monetarily and from the point of view of safety. In most cases, these failures can be related to faults, which could be either catastrophic or parametric (drift from nominal value) [1]. Systems have multiple simultaneously degrading components and because of their interactions and collateral damage, system RUL computation is more than a simple combination of individual component RULs. Circuit failure prevention requires methods for the early detection and isolation of faults and prediction of RUL of the failing circuit [1]. Most studies in literature focus on these methods but only for individual components. While useful, this misses the bigger picture, because system-level RUL is of greater interest to Varun Khemani. This is an open-access article distributed under the terms of the Creative Commons Attribution 3.0 United States License, which permits unrestricted use, distribution, and reproduction in any medium, provided the original author and source are credited. maintainers of these systems. Fault diagnostics and prognostics in analog circuits are made challenging by the presence of component tolerances, the complex nature of the fault mechanisms [1]. For system-level prognostics, [2] setup system level prognostics by using a particle filtering based approach where they assume that the system state function and the measurement function is known. They use an example of a simple full wave rectifier. [3] use successive refinement of circuit's response to a sweep signal, to extract features for fault diagnosis. The fault diagnostics problem is solved as a pattern recognition problem using kernel methods with the assumption that a sweep signal can excite a circuit for feature extraction of faults being pivotal. We propose a method a new fusion prognostics based approach that has no such limiting assumptions.

\section{EXPECTED CONTRIBUTIONS}

As mentioned previously, the proposed approach has no limitations except for the assumption that the circuit can be modeled in a modeling software like PSpice. In most published studies, interactions between degrading components are ignored. This study will show that such an exclusion could result in potentially disastrous results. We also tackle the issue of generating training data in a systematic way to have sufficient coverage of parametric space. We also try to improve the estimation of two-way and higher order interactions by making the estimation with as fewer degrees of freedom as possible. Finally, we try to improve state of the art deep learning architectures and try to come up with new architectures, which work best for circuit fault diagnosis and prognosis. This represents a comprehensive PHM framework that is universally applicable.

\section{RESEARCH PLAN}

Task 1: Model the circuit in a modeling software using PSpice

Task 2: Conduct a FMMEA (Failure Modes, Mechanisms, and Effects Analysis) on the circuit components to identify the list of component parameters in PSpice that are indicative of component's dominant degradation mechanism(s).

Task 3: Conduct a design of experiment involving the selected parameters of every component, to ascertain the components whose degradation has a significant impact on the circuit output.

Task 4: For the significant components only, generate the training data by varying them in a one-at-a-time setting where other components are held constant. 
Task 5: Experiment with training of different deep learning architectures in an effort to improve diagnostic accuracy. Task 6: Conduct a thorough literature search and along with previous CALCE experience, collect physics of failure models for the different failure modes of the different significant components.

Task 7: Use the best performing architecture from task 5 and replace the final layer by a regression layer. Perform training again to predict component degradation. Use prediction and the failure models from task 6 to trend the component to failure and arrive at the circuit RUL.

As a proof of concept, we consider an example of a double switch forward converter circuit which has 17 components. Additionally, we also compare results to a 130 component circuit that we worked on but cannot share the details because of confidentiality concerns. We refer to these circuits as Circuit A and Circuit B respectively. PSpice has multiple parameters for each component for simulation of its behavior. The challenge is to sift through these parameters to figure out how different failure mechanisms can be simulated through a change in these parameters. For example, 14 parameters for diodes, 6 parameters for capacitors, 40 for bipolar junction transistors and 50 parameters for MOSFETs. FMMEA enables the identification and prioritization of failure modes and mechanisms for the system components. Based on the dominant failure mechanisms identified in the FMMEA, we can select only those PSpice parameters that are indicative of the component degradation via the dominant mechanisms. Because of the consideration of multiple parameters for some components, we now have 25 parameters for Circuit A and 164 parameters for Circuit B.

\subsection{Work Performed}

Parametric space of a system is the entire region where the system operates or breaks down i.e. it is the conjunction of all the components of a circuit from their open circuit to the nominal to their short circuit condition. It might not be possible to generate training data in regions where it is difficult to carry out experiments. For example, in an analog circuit, it is usually difficult/not possible physically experiment with active components and hence training data cannot be generated for the same. If a simulation model can be developed in PSpice, better coverage of the design space of the parametric space can be achieved. In order to optimally explore the parametric space, a special experiment called space-filling design is used. There are various construction methods for space-filling designs but we use the Maximum Entropy designs. These designs maximize the Shannon information [4] of an experiment.

$$
R_{i j}=\exp \left(-\sum_{k} \theta_{k}\left(x_{i k}-x_{j k}\right)^{2}\right) \mathrm{R}_{\mathrm{ij}} \text { is the correlation of }
$$

response values at two different design points, xi and xj. For the same number of runs (simulations), Maximum Entropy designs provide a higher coverage of the parametric space as compared to fractional factorial designs. The number of runs (simulations) required to estimate two way interactions among $\mathrm{X}$ parameters is $\left(\begin{array}{l}X \\ 2\end{array}\right)$. Hence, for estimating main effects and two-way interactions we would require, for circuit A, 325 runs (simulations), and for circuit B, 13530 runs (simulations). Because of this excessive number of runs (simulations) and the associated simulation time in PSpice, we estimate only the main effects from the initial spacefilling design. The number of significant main effects are 10 for Circuit A and 46 for Circuit B. Next, we use the concept of supersaturated designs where we can estimate more terms than we have the degrees of freedom for [5]. A general principal in supersaturated designs is that the number of terms that we have to estimate should not be more than twice the number of runs (simulations) we have [5]. Hence, we can estimate all two-way interactions using just 150 additional runs (simulations) for Circuit $\mathrm{A}$ and 6680 additional runs (simulations) for circuit B. We estimated that 30 parameters (10 main effects and 20 interactions) were significant for Circuit A and 138 parameters (46 main effects and 92 twoway interactions) were significant for Circuit B. Even though they are statistically significant, they might not cause the output waveform to degrade beyond predefined limits. We do a one-at-a-time study on these significant parameters by varying each parameter from $-75 \%$ to $+75 \%$ of the nominal while other parameters are held constant at their nominal values. Now, we can reduce the set to only those parameters that cause the waveform to go outside at least one of the thresholds. This leads to fewer significant parameters, 20 total parameters (5 main effects and 15 two-way interactions) for circuit $\mathrm{A}$ and 36 total parameters (4 main effects and 32 two-way interactions) for circuit B and hence fewer classes of diagnosis for the next step. However, more important are the trends (Figure 1), which show that, R5, by itself causes waveform peak to fall below the threshold when it degrades by $135 \%$ of its nominal value. However, when R27 and Vs2 are both degrading together, they only need to degrade by $115 \%$ to cause the waveform peak to go below the threshold. Similar patterns are observed by comparing the degradation of other single components and their interactions with other degrading components for Circuit A and even for circuit B. Hence, ignoring interactions of degrading components could have potentially disastrous effects. Next, the training data is generated for the deep learning networks using the same one-at-a-time study format. We start by generating 5000 samples per class, taking advantage of the entire waveform for classification purposes and not just the output voltage peak. When circuit parameters are far from their nominal values, PSpice sometimes runs into convergence issues. When this happens, PSpice increases the sampling rate, which causes the output file sizes to be of different lengths across different training samples even in the same class. Instead of undersampling some training samples and oversampling others to come to a constant length for every training sample, we plot the entire voltage series without changing its sampling rate and convert the plot into an image. This ensures that we do not lose out on any waveform features due to undersampling. These 
images can now be used as an input to deep neural networks, for image classification (fault diagnosis).

Since deep learning is not an exact science, we experiment with different deep learning architectures, in order to ascertain the architecture that provides the best classification accuracy. The architectures we consider are VGG16 [6], GoogLeNet [7] and ResNet50 [8]. We also experiment with different sizes and number of convolution layers, different activation functions, removing pooling layers, different settings for the backpropagation algorithms etc. We obtain the following result for Circuit A. For circuit B, the classification accuracies are poor even in the case of Renset50 because the within-class variance is greater than

\begin{tabular}{|l|l|l|}
\hline Method & $\begin{array}{l}\text { Classification } \\
\text { Accuracy }\end{array}$ & Time \\
\hline VGG16 & $94.19 \%$ & 5 hours \\
\hline GoogLeNet & $97.39 \%$ & 8 hours \\
\hline Resnet 50 & $98.79 \%$ & 10 hours \\
\hline
\end{tabular}

the between-class variance.

\section{Remaining Work}

a) Current research in statistics in restricted to two-way interaction screening in high dimension experiments [9]. However, using advanced analysis techniques like regularized regression to solve the ill-poised problem we hope to estimate two-way and higher order interactions using fewer runs (simulations) as a part of remaining work.

b) Develop a library of physics of failure models for different failure mechanism for different components.

c) Currently, there are many pixels being wasted in the square image of the waveform where there is no waveform. Therefore, we intend to develop a new representation for the waveform to reduce pixel wastage.

d) We will try to improve state of the art deep learning architectures and try to come up with new architectures, which work best for circuit fault diagnosis and prognosis.

\section{Conclusion}

We demonstrate a comprehensive PHM framework that can be applied to any circuit even if it does not have operational data. Using design of experiment enables us to study of interaction among degrading components. This is particularly significant as most studies in literature focus on degradation of individual components and not their interaction with other degrading components. Risks of ignoring interactions are demonstrated and techniques to explore higher order interactions are proposed. These results can help circuit designers improve their circuits. Additionally, we take advantage of the benefit in using fusion-based prognostics as opposed to a purely data-driven approach. This benefit is analogous to the benefit accrued in using prior information as opposed to an uninformative prior in Bayesian analysis.
[1] M. A. Alam, M. H. Azarian, M. Osterman, and M. Pecht, "Prognostics of failures in embedded planar capacitors using model-based and data-driven approaches," J. Intell. Mater. Syst. Struct., vol. 22, pp. 1293-1304, 2011.

[2] Khorasgani, H., Biswas, G., \& Sankararaman, S. (2016). Methodologies for system-level remaining useful life prediction. Reliability Engineering \& System Safety

[3] Vasan, A. S. S., Long, B., \& Pecht, M. (2013). Diagnostics and prognostics method for analog electronic circuits. IEEE Transactions on Industrial Electronics, 60(11), 5277-5291.

[4] Shewry, M.C. and Wynn, H.P. (1987), "Maximum Entropy Sampling," Journal of Applied Statistics 14, 165

[5] Holcomb, D. R., Montgomery, D. C., \& Carlyle, W. M. (2003). Analysis of supersaturated designs. Journal of Quality Technology, 35(1), 13.

[6] K. Simonyan and A. Zisserman, "Very deep convolutional networks for large-scale image recognition,"arXiv preprint arXiv:1409.1556, 2014.

[7] Szegedy, C.; Liu, W.; Jia, Y.; Sermanet, P.; Reed, S.; Anguelov, D.; Erhan, D.; Vanhoucke, V.; and Rabinovich, A. 2014. Going deeper with convolutions. CoRRabs/1409.4842.

[8] He, K., Zhang, X., Ren, S., \& Sun, J. (2016). Deep residual learning for image recognition. In Proceedings of the IEEE conference on computer vision and pattern recognition (pp. 770-778).

[9] Hao, N., \& Zhang, H. H. (2014). Interaction screening for ultrahigh-dimensional data. Journal of the American Statistical Association, 109(507), 1285-1301.

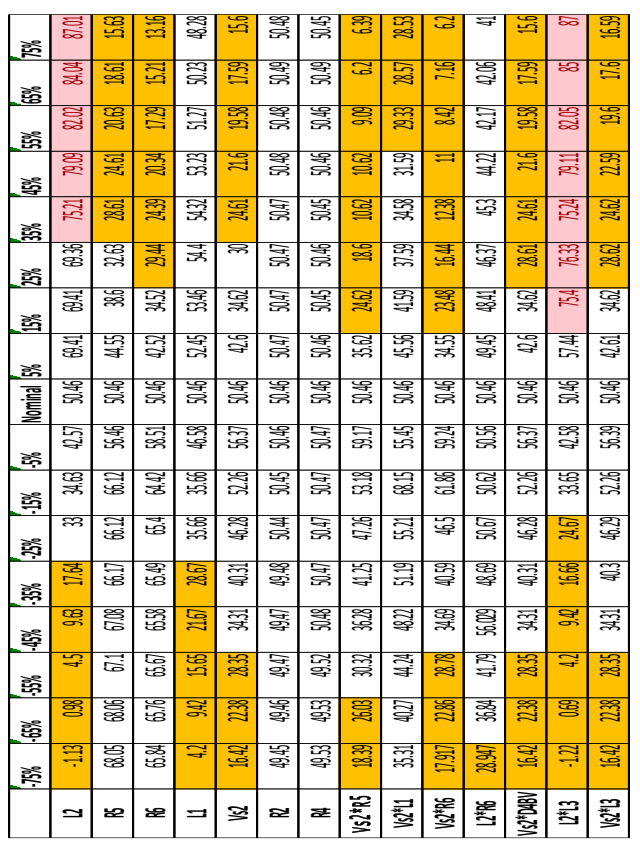

\section{REFERENCES}

